\title{
Genetically driven adiposity traits increase the risk of coronary artery disease independent of blood pressure, dyslipidaemia, glycaemic traits
}

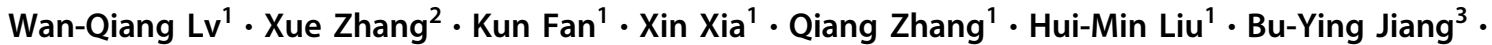 \\ Wei-Dong Zhang ${ }^{1} \cdot$ Hong-Wen Deng ${ }^{1,4}$
}

Received: 25 June 2017 / Revised: 30 April 2018 / Accepted: 8 May 2018 / Published online: 11 June 2018

(c) European Society of Human Genetics 2018

\begin{abstract}
Adiposity has been associated with the risk of coronary artery disease (CAD) in observational studies, but their association may differ according to specific characteristics of studies. In Mendelian randomization (MR) analyses, genetic variants are used as instrumental variables (IVs) of exposures to examine causal effects to overcome confounding factors and reverse causation. We performed MR analyses for adiposity $(n=322,154)$ on risk of CAD $(60,801$ cases and 123,504 controls) based on the currently largest genome-wide association studies. The estimated associations between adiposity traits and CAD were calculated by an inverse-variance weighted method with and without excluding the IVs, which are associated with the well-known risk factors of CAD. Genetic variants are identified to be associated with the well-known risk factors of CAD by a cross-phenotype meta-analysis method. Our results furnished strong evidence for a causal role of adiposity in risk of CAD, with the odds ratios (ORs) for CAD being 1.53 (95\% CI 1.36-1.72) for body mass index (BMI), 1.48 (1.20-1.96) for waist-hip ratio (WHR), and 1.34 (1.07-1.59) for WHR adjusted for BMI (WHRadjBMI), respectively. After excluding mediators-associated IVs from the MR analyses, the corresponding ORs were 1.46 (1.28-1.67) for BMI, 1.39 (1.01-1.93) for WHR, and 1.38 (1.04-1.84) for WHRadjBMI, respectively. Furthermore, our results suggested that central adiposity and general adiposity might pose a similar risk for CAD. In summary, our data supported that genetically driven adiposity traits imposed the risk of CAD independent of blood pressure, dyslipidaemia, glycaemic traits, and type 2 diabetes.
\end{abstract}

These authors contributed equally: Wan-Qiang Lv, Xue Zhang, Kun Fan, Xin Xia

Electronic supplementary material The online version of this article (https://doi.org/10.1038/s41431-018-0180-9) contains supplementary material, which is available to authorized users.

Wei-Dong Zhang imooni@163.com

$\triangle$ Hong-Wen Deng hdeng2@tulane.edu

1 College of Public Health, Zhengzhou University, ZhengzhouNO.100 Kexue Road, High-Tech Development Zone of StatesPeople's Republic of China

2 Department of Geriatrics, Renmin Hospital of Wuhan University, Wuhan 430060 Hubei Province, People's Republic of China

3 Henan Health Cadre College, ZhengzhouNO.149 Huayuan Road, Jinshui District, People's Republic of China

4 Center for Genomics and Bioinformatics, School of Public Health and Tropical Medicine, Tulane University, New Orleans, LA 70112, USA

\section{Introduction}

Obesity is a chronic metabolic disorder, mainly characterized by excessive body fat. According to National Health and Nutrition Examination Survey 2013 to 2014, the prevalence of obesity among US adults increased from 1999 to 2000 through 2013 to 2014 from 30.5 to $37.7 \%$ [1, 2], making it a major contributor to the rise of prevalence of coronary artery disease (CAD) [3].

Epidemiological studies have identified obesity as an independent risk factor of cardiovascular disease. A systematic review and meta-analysis of 37 studies showed that high childhood body mass index (BMI) was associated with an increased incidence of adult CAD (Odds ratio-OR: $1.20,95 \%$ confidence interval-CI: $1.10-1.31$ ) [4]. Similarly, a large meta-analysis showed that obese subjects had a significantly greater risk of CAD (relative risk-RR: 1.81, 95\% CI: 1.56-2.10) after adjustment for age, sex, physical activities, and smoking [5]. Despite the association between obesity with an increased occurrence of CAD, studies have 
reported the obesity paradox phenomenon, in which overweight and obese patients with established CAD have better clinical outcomes than normal weight patients [6, 7]. Inconsistent findings may be partly a result of different sample sizes and length of follow-up periods for these studies, or the inclusion of individuals with different clinical characteristics [4-7].

Studies have reported that the impact of adiposity on CAD risk is determined by the degree to which fat accumulates as well as where it accumulates [8]. When determining adiposity in research and clinical diagnosis, BMI was widely used. However, it may overestimate body fat in people who are very muscular and underestimate body fat in those who have lost muscle mass [9]. Increasing studies suggested that the accumulation of abdominal adipose tissue appears to be an independent risk predictor of CAD, while the accumulation of abdominal adipose tissue (measured by waist-hip ratio (WHR)) is associated with greater CAD risk [10, 11]. Another recent study reported that measures of adiposity (BMI, WHR) were related to CAD, but did not improve risk discrimination when adjusted for the well-known risk factors of $\mathrm{CAD}$, including diabetes mellitus, blood pressure, and blood lipids [12]. However, a publication showed that the association between adiposity and CAD was attenuated to null apart from blood pressure, cholesterol level, and blood glucose level [13].

In observational studies, adiposity has been associated with CAD [14]. However, unmeasured factors might confound observational studies that link BMI and WHR with CAD [15]. Moreover, adiposity was often based on selfreported or measured values of weight/BMI and height, which tend to be under-reported and over-reported, respectively [16]. Additionally, reverse causality could similarly lead to a statistically robust but non-causal relationship. For example, individuals with subclinical CAD might develop abdominal adiposity because of an inability to exercise. It is unclear whether the relationship found between adiposity traits and CAD in observational studies is causal. Mendelian randomization (MR) method may help to investigate the possible causal associations between adiposity traits and CAD as it could eliminate potential reverse causality and reduce confounding bias [17, 18]. Several recent MR studies have estimated the effect of adiposity traits on CAD risk [19-21]. However, these studies included various numbers of adiposity-associated genetic variants that are associated with the well-known risk factors of $\mathrm{CAD}$, thereby allowing for the possibility that such variants have effects on the risk factors of $\mathrm{CAD}$, thus the relationship between adiposity and CAD may be mediated by these factors. Some studies just estimated the association of WHR adjusted for BMI with CAD [19, 20]. However, the collider bias might exist, whereby the BMI adjustment biases the causal estimate due to the collinearity with WHR and its likely mediating or confounding role in the association of WHR and CAD.

Here we applied MR analyses to quantify and contrast the association of adiposity traits on the risk of CAD based on summary statistics from the largest published genomewide association studies (GWAS) [22, 23]. In our study, we applied a cross-phenotype meta-analysis method to identify such variants that are associated with the well-known risk factors of CAD, including blood pressure, dyslipidaemia, glycemic traits and type 2 diabetes. With excluding the mediators-associated variants from MR analyses, we could specifically investigate the estimated associations between adiposity traits and CAD independent of the well-known risk factors of CAD. We investigated the association of WHR both adjusted and unadjusted for BMI with CAD to discuss whether the collider bias influences the results.

\section{Materials and Methods}

\section{Data sources}

The summarized data sets for adiposity traits were obtained from the largest meta-GWAS performed by the Genetic Investigation of Anthropometric Traits (GIANT) Consortium and downloaded from the site: http://portals.broa dinstitute.org/collaboration/giant/index.php/GIANT_consor tium_data_files. Effect estimates of SNPs associated with BMI were obtained from a two-stage meta-analysis to identify BMI-associated loci in European adults $(n=$ $322,154)$, where BMI was adjusted for age, age squared, and any necessary study-specific covariates in a linear regression model [22]. Effect estimates of SNPs associated with WHR and WHR adjusted for BMI (WHRadjBMI) were obtained from meta-GWAS of waist and hip circumference-related traits in European individuals $(n=$ 224,459) [23]. Estimates of the effects of these traitassociated SNPs on risk of CAD were obtained from the meta-GWAS of mainly European descent $(60,801$ CAD cases and 123,504 controls), conducted by the Coronary ARtery DIsease Genome wide Replication and Metaanalysis (CARDIoGRAM) plus the Coronary Artery Disease (C4D) Genetics consortium (CARDIoGRAMplusC4D) [24] and downloaded from the site: http://www.cardiogra mplusc4d.org/data-downloads. Further details of the GWAS samples and methods employed within each group were presented in the original references $[22,23,25]$.

\section{SNP selection}

An important assumption of MR analyses is that the SNP was associated with the exposure (e.g., adiposity traits). Several steps were involved to select the adiposity- 
associated SNPs as instrumental variables (IVs) for analysis. First, we identified the adiposity-associated SNPs that achieved the standard threshold of genome-wide significance $\left(p<5 \times 10^{-8}\right)$ in the GIANT consortium, respectively. Then for these selected SNPs, we applied the linkage disequilibrium (LD)-based pruning method to remove the large correlations between pairs of variants. The data sets were pruned using the HapMap3 genotypes of the corresponding ethnicity references. SNPs were removed from our analysis if their measured LD had an $r^{2}>0.001$. We retained the SNP that was most strongly associated with adiposity traits by $p$ value when two or more SNPs were in LD. Last, we gathered summary-level results for each SNP from the CAD GWAS data and excluded the SNPs that were associated with CAD (For the SNPs, the $p$ values from summary-level results were smaller than $5 \times 10^{-8}$ in the CAD GWAS). The summary associations of candidate IVs were harmonized as described [26]. Overall, we identified the SNPs as IVs, which are associated with adiposity traits and are not associated with the outcome (e.g., CAD).

\section{Pleiotropic variants detected by cross-phenotype meta-analysis}

Accumulative evidence indicated that pleiotropic effect exists, a single gene or SNP is associated with more than two distinct phenotypes [27]. Especially, it is relevant for studies conditioning on the pleiotropic effect that will lead to the inclusion of adiposity-associated IVs, which are associated with risk factors that may increase or protect against CAD. Additional exclusion of genetic variants that are associated with the well-known risk factors of CAD could help us investigate the association between adiposity and $\mathrm{CAD}$ independent of these risk factors of CAD, including blood pressure, dyslipidaemia, glycaemic traits and type 2 diabetes. Thus, the cross-phenotype meta-analysis (CPMA) method was used to detect potential pleiotropic effect between the adiposity traits with the other wellknown risk factors of CAD [28]. This method could identify any SNP for which there is statistical evidence of potential pleiotropic effects on possible mediators, ignoring the strength or the direction of genetic effect. Previous studies reported that the well-known risk factors may increase or protect against CAD, we included cohort-specific summarylevel results from the currently largest GWAS metaanalyses for each trait, where such data were publicly available: (1) blood lipids (low-density lipoprotein cholesterol (LDL), high-density lipoprotein cholesterol (HDL), and triglycerides (TG), total cholesterol (TC)) [29], (2) systolic and diastolic blood pressure [30], (3) glycemic traits (fasting glucose, $2 \mathrm{~h}$ glucose) [31], (4) type 2 diabetes [32]. In our study, we applied the restrictive threshold of pleiotropic associations $(p<0.01)$ on possible mediators.
Meta-analysis literature revealed MR-Egger regression to be a valuable means of testing for directional pleiotropy [33]. Thus, MR-Egger method was applied for visual inspection of pleiotropic effects of the included SNPs. If an intercept of MR-Egger regression test was not distinct from the origin, it indicated that the included SNPs were free of pleiotropic effect.

\section{Statistical analyses}

We used an inverse-variance weighted (IVW) meta-analysis of ratio estimates method by weighting the genetic effect estimate of each SNP on CAD by its effect on adiposity trait [34]. This method was reported to have equivalent statistical power with two-stage least squares method and successfully applied to various diseases [35-37]. In this method, SNP $j$ $(j=1, \ldots, \mathrm{j})$ was associated with an observed $X_{\mathrm{j}}$ mean change in the exposure per additional variant allele with standard error $\sigma_{X_{\mathrm{j}}}$ and an observed $Y_{\mathrm{j}}$ mean change in the outcome per allele with standard error $\sigma_{Y_{j}}$. For $\operatorname{SNP} j$, the causal effect estimate of the exposure on the outcome is equivalent to the ratio of $Y_{j} / X_{j}$. The standard error of the ratio can be calculated as the ratio of $\sigma_{Y_{j}} / X_{j}$. When putting each SNP into a fixed-effect meta-analysis model, the causal effect estimate of the exposure on the outcome can be calculated as the ratio

$$
\widehat{\beta}=\frac{\sum_{j} X_{j} Y_{j} \sigma_{Y_{j}}^{-2}}{\sum_{j} X_{j}^{2} \sigma_{Y_{j}}^{-2}}
$$

The standard error of the estimate is approximated by

$$
\operatorname{se}(\hat{\beta})=\sqrt{1 / \sum_{j} X_{j}^{2} \sigma_{Y_{j}}^{-2}}
$$

Heterogeneity of the overall identified SNPs for adiposity traits was assessed using the $Q$ statistic and reported as a $p$ value of heterogeneity. Cook's distance was used to estimate heterogeneity of each SNP associated with adiposity traits.

\section{Results}

\section{Instrument Validation}

Where possible, we tested the following three fundamental MR assumptions to ensure the validity of IVs [38]: (i) SNPs used as IVs must be associated with the exposure of interest (e.g., BMI, WHR, WHRadjBMI); (ii) SNPs must not be associated with potential confounders; and (iii) SNPs must be only associated with the outcome variable (CAD) via the exposure of interest. 
Table 1 Instrumental variable analyses of the causal effects of adiposity traits on risk of CAD

\begin{tabular}{|c|c|c|c|c|c|c|c|}
\hline \multirow[t]{2}{*}{ Exposure } & \multirow[t]{2}{*}{$\mathrm{N}$} & \multicolumn{3}{|c|}{ IVW results } & \multicolumn{2}{|c|}{$\begin{array}{l}\text { MR-Egger } \\
\text { regression }\end{array}$} & \multirow{2}{*}{$\begin{array}{l}\text { Heterogeneity } \\
p \text { value }\end{array}$} \\
\hline & & OR & $95 \% \mathrm{CI}$ & $p$ value & Intercept & $p$ value & \\
\hline BMI & 68 & 1.53 & $1.36-1.72$ & $5.1 \times 10^{-12}$ & -0.01 & 0.44 & 0.0009 \\
\hline WHR & 28 & 1.48 & $1.20-1.67$ & 0.006 & -0.15 & 0.91 & $1.7 \times 10^{-6}$ \\
\hline WHRadjBMI & 36 & 1.34 & $1.07-1.59$ & 0.009 & -0.03 & 0.70 & $5.6 \times 10^{-6}$ \\
\hline $\mathrm{BMI}^{\mathrm{a}}$ & 46 & 1.46 & $1.28-1.67$ & $2.9 \times 10^{-8}$ & 0.00 & 0.80 & 0.30 \\
\hline $\mathrm{WHR}^{\mathrm{a}}$ & 12 & 1.39 & $1.01-1.93$ & 0.04 & 0.02 & 0.27 & 0.09 \\
\hline WHRadjBMI $^{\mathrm{a}}$ & 16 & 1.38 & $1.04-1.84$ & 0.03 & 0.02 & 0.33 & 0.61 \\
\hline
\end{tabular}

Estimates for the effect of adiposity traits with coronary artery disease were derived from Mendelian randomization analyses

$I V W$ inverse-variance weighted method, BMI body mass index, WHR waist-hip ratio, WHRadjBMI waist-hip ratio adjusted for BMI, $O R$ odds ratio, $C I$ confidence interval, $N$ numbers of included instrumental variables in the Mendelian randomization analyses

${ }^{a}$ Indicates that the results were derived from the sensitivity analyses, excluding the pleiotropic variants which were associated with intermediate factors by the cross-phenotype meta-analysis
For assumption (i), several steps were conducted to ensure that the included SNPs are associated with the exposure of interest, thus validating this assumption. Additionally, population stratification is a potential source of bias, the GWAS used in our study included only individuals of European descent and thus selected to control the bias. Overall, we identified 68 BMI SNPs, 28 WHR SNPs and 36 WHRadjBMI SNPs as IVs for the exposure, respectively. Details of individual SNPs were given in Supplemental Tables 1-3. There was no overlap between BMI SNPs and WHR SNPs, WHRadjBMI SNPs. For assumption (ii), MR-Egger regression was used to investigate whether the association was biased by any unbalanced horizontal pleiotropy or not. The CPMA method was used to identify the IVs which are associated with the well-known risk factors of CAD, so we can assess the vertical pleiotropy between the IVs and these factors in our study. Briefly, we included these factors as mediators and considered the restrictive pleiotropic threshold of pleiotropic associations $(p<0.01)$. Details of pleiotropic associations for each SNP across potential mediators were described in Supplementary Tables 4-6. At last, MR-Egger regression was performed to test and correct for bias as a result of directional pleiotropic effect. According to the results of MR-Egger regression test, the estimated intercept terms were all centered at the origin which indicated that genetic pleiotropic effect had not influenced the causal effect estimates, as shown in Table 1. For assumption (iii), it was difficult to test directly. However, SNPs used as IVs had been identified to be associated with the exposure and yet had no obvious biological link to other potential confounders (smoking status and educational attainment).

\section{Instrumental variable analysis}

\section{$B M I$ on the risk of $C A D$}

Detailed information on the included SNPs of BMI was shown in the Supplementary Table 1. According to the results of the MR-Egger regression test, the intercept was near zero (Intercept: $-0.004, p=0.440$ ), indicating that the causal estimate of BMI with the risk of CAD was not biased by any unbalanced directional pleiotropy. In the IV analysis using a polygenic instrument of 68 BMI SNPs, we found a positive association of BMI with the risk of CAD from the IVW results (OR: 1.53, per standard deviation (SD) increase of BMI, 95\% CI: $1.36-1.72, p=$ $\left.5.1 \times 10^{-12}\right)$. However, the heterogeneity test supported heterogeneity in the IVs of BMI $(p=0.0009)$. As suggested in the study by Jack Bowden and coworkers [38], we conducted a sensitivity analysis using a median-weighted MR method to take into account the heterogeneity in the IVs. The results from the weighted median were consistent with the IVW results $(\mathrm{OR}=1.50$, per $\mathrm{SD}$ increase of $\mathrm{BMI}$, $95 \%$ CI: $\left.1.30-1.75, p=1.3 \times 10^{-8}\right)$. Next, we investigated each of the BMI SNPs for potential pleiotropic effects. At a conservative threshold of the CPMA method $(p<0.01)$, there were 22 pleiotropic SNPs, resulting in 46 BMI SNPs being treated as IVs which were not associated with the analyzed known mediators (Supplementary Table 4). Using the IVs of 46 BMI-associated SNPs, one-SD genetically elevated BMI was associated with a $46 \%$ increase in odds of CAD $(\mathrm{OR}=1.46$, per SD increase of BMI, 95\% CI: $1.28-1.67, p=2.9 \times 10^{-8}$, heterogeneity $p=0.30)($ Table 1$)$. 


\section{WHR on the risk of CAD}

Detailed information on the included SNPs for WHR and WHRadjBMI was shown in Supplementary Tables 2 and 3, respectively. On the basis of the results of the MR-Egger regression test, the estimated causal effect of WHR on the risk of CAD was not biased by any unbalanced horizontal pleiotropy (Intercept for WHR: $-0.002, p=0.913$; intercept for WHRadjBMI: $-0.005, p=0.702$ ). In the IV analyses ignoring the SNPs that might be associated with possible mediators, our results suggested a causal effect of WHR on the risk of CAD $(\mathrm{OR}=1.48$, per SD increase of WHR, 95\% CI: $1.20-1.96, p=0.006$, heterogeneity $p=$ $1.7 \times 10^{-6}$; OR $=1.34$, per SD increase of WHRadjBMI, $95 \%$ CI: $1.07-1.59, p=0.009$, heterogeneity $p=5.6 \times 10^{-6}$ ). When taking into account the heterogeneity in the IVs, the results from the weighted median was consistent with the IVW results $(\mathrm{OR}=1.44$, per SD increase of WHR, 95\% CI: $1.11-1.87, p=0.005$; $\mathrm{OR}=1.32$, per $\mathrm{SD}$ increase of WHRadjBMI, 95\% CI: $1.08-1.62, p=0.009)$. After excluding the pleiotropic SNPs identified by CPMA method (Supplementary Tables 5 and 6), we showed that the association of increased WHR and CAD was preserved $(\mathrm{OR}=1.39$, per SD increase of WHR, 95\% CI: 1.01-1.93, $p=0.04$, heterogeneity $p=0.09 ; \mathrm{OR}=1.38$, per $\mathrm{SD}$ increase of WHRadjBMI, 95\% CI: $1.04-1.84, p=0.03$, heterogeneity $p=0.61$ ).

\section{Discussion}

The present study utilized an MR design applied to the studies within the GIANT consortium to address the potential causal role of central adiposity (measured by WHR and WHRadjBMI) and general adiposity (measured by $\mathrm{BMI}$ ) on the risk of CAD. Our MR investigation confirmed that genetically driven adiposity traits, determined by adiposity-associated genetic variants, could significantly increase in odds of CAD. After excluding the variants which are associated with the major known risk factors of $\mathrm{CAD}$, there was still a significantly increased risk of CAD that is independent of the analyzed blood pressure, dyslipidaemia, glycaemic traits and type 2 diabetes. Additionally, central adiposity and general adiposity might pose a similar risk for CAD ( $p$ values from the $t$ test $>0.05$ ).

Some MR analyses have been applied to test the association of adiposity with CAD. However, these studies mainly focused on BMI as an exposure proxy solely for adiposity $[21,39,40]$, while our study extended to other measures of adiposity and fat distribution as exposure proxies, including WHR with and without adjustment for BMI. Moreover, the earlier studies included smaller sample sizes, leading to weaker IVs due to the limited numbers of
SNPs identified as IVs for MR analyses [21, 39, 40].Our results were in line with two recent MR studies based on the analysis of both summary statistics and individual-level data $[19,20]$. Moreover, we quantified and contrasted causal associations of central adiposity and general adiposity with CAD.

In line with our results, cohort investigations have demonstrated that adiposity is related to CAD apart from its association with the well-known risk factors, including blood pressure, dyslipidaemia and glycaemic traits [41, 42]. Several mechanisms could underlie an effect of adiposity on CAD independent of the well-known risk factors. In addition to these well-known risk factors, other consequences of adiposity are also likely to contribute to the risk of CAD, including a state of low-grade inflammation [43, 44], endothelial dysfunction [45], impaired endothelial vasodilatory responses [46]. In anatomical structure, obese individuals are associated with increases in total blood volume, stroke volume, and cardiac output which may lead to abnormal left ventricle remodeling and left ventricle hypertrophy over time, and withdrawal of vagal activity and sympathetic predominance may lead to reduced heart rate variability, all of which combine may further increase the likelihood of developing CAD [47].

Our results may allow several conclusions. First, central obesity has a causal effect on CAD that is independent of BMI. This finding demonstrates the potential of MR approaches for investigating highly correlated adiposity traits that have proved challenging to disentangle in observational studies [48]. Second, it is not only the volume of adiposity, but also its location had the impact on the risk of CAD that is independent of total fat. For example, at a given BMI, there is considerable inter-individual variation in the amount of visceral fat, which shows associations with diseases [49]. Third, efforts to quantify the effect of obesity on the burden of CVD should include multiple measurements of body fat distribution to avoid underestimating the true burden of obesity on health [50].

This study has several strengths. First, independent SNPs were used as IVs to investigate the estimates effect of central and general obesity on CAD. Second, the CPMA method was applied to identify the variants which are associated with the well-known risk factors of CAD, so that we could investigate the estimated effects of adiposity traits on CAD independent of dyslipidaemia, blood pressure, and glycemic traits and type 2 diabetes in the present study. This study may also have several potential limitations. First, although several methods were used in an attempt to rule out pleiotropy, it is possible that the results represent a shared genetic basis between obesity and CAD rather than a causal relationship [51]. Second, the association of adiposity traits with CAD was assessed in linear regression model, but a log-linear association may not exist between 
adiposity traits and cardiometabolic traits in observational studies [52]. However, when a BMI value is greater than 25 $\mathrm{kg} / \mathrm{m}^{2}$, the association has been reported to be linear [53] and therefore our findings are primarily applicable to those individuals. Third, this analysis was only included individuals of European descent, the association of genetically determined adiposity traits with CAD may differ by ethnicity or genetic ancestry.

In conclusion, our study supported evidence for a causal role of both central and general adiposity in risk of CAD independent of the well-known risk factors of CAD, including dyslipidaemia, blood pressure, and glycemic traits and type 2 diabetes. Furthermore, our results suggested that central adiposity separated from general adiposity and general adiposity might pose a similar risk for CAD. Our findings suggested that the impact of adiposity on risk of CAD is determined by the degree to which fat accumulates as well as where it accumulates. In clinic in practice, physicians should therefore pay attention to the potential effects of different obesity-related traits with CAD.

Acknowledgements We appreciate the support from Zhengzhou University in providing necessary support for this collaborative project. H-WD was partially supported by grants from NIH (AR069055, U19 AG055373, R01 MH104680, R01AR059781, U19AG055373 and P20GM109036).

Author contribution W-QL, XZ, XX, and KF all as the first author performed the data analysis and wrote the manuscript. QZ, H-ML, and $\mathrm{B}-\mathrm{YJ}$ provided advice and suggestions during the data analysis process. W-DZ and H-WD conceived and initiated this project, oversaw the implementation of the statistical method, and revised/finalized the manuscript.

\section{Compliance with ethical standards}

Conflict of interest The authors declare that they have no conflict of interest.

\section{References}

1. Flegal KM, Kruszon-Moran D, Carroll MD, Fryar CD, Ogden CL. Trends in obesity among adults in the United States, 2005 to 2014. JAMA. 2016;315:2284-91.

2. Ogden CL, Carroll MD, Fryar CD, Flegal KM: Prevalence of obesity among adults and youth: United States, 2011-2014. NCHS data brief 2015:219:1-8.

3. Klein S, Burke LE, Bray GA, et al. Clinical implications of obesity with specific focus on cardiovascular disease: a statement for professionals from the American Heart Association Council on Nutrition, Physical Activity, and Metabolism: endorsed by the American College of Cardiology Foundation. Circulation. 2004;110:2952-67.

4. Llewellyn A, Simmonds M, Owen CG, Woolacott N. Childhood obesity as a predictor of morbidity in adulthood: a systematic review and meta-analysis. Obes Rev. 2016;17:56-67.

5. Bogers RP, Bemelmans WJ, Hoogenveen RT, et al. Association of overweight with increased risk of coronary heart disease partly independent of blood pressure and cholesterol levels: a meta-analysis of 21 cohort studies including more than 300000 persons. Arch Intern Med. 2007;167:1720-8.

6. Lin GM, Li YH, Yin WH, et al. The obesity-mortality paradox in patients with heart failure in taiwan and a collaborative metaanalysis for East Asian patients. Am J Cardiol. 2016;118:1011-8.

7. Carnethon MR, De Chavez PJ, Biggs ML, et al. Association of weight status with mortality in adults with incident diabetes. JAMA. 2012;308:581-90.

8. Bjorntorp P. Metabolic implications of body fat distribution. Diabetes Care. 1991;14:1132-43.

9. Bahat G, Tufan F, Saka B, et al. Which body mass index (BMI) is better in the elderly for functional status? Arch Gerontol Geriatr. 2012;54:78-81.

10. Rashiti P, Elezi S, Behluli I, Mucaj S. Relationship of plasma adiponectin and waist-hip ratio with coronary artery disease. Med Arch. 2016;70:413-8.

11. Carmienke S, Freitag MH, Pischon $\mathrm{T}$, et al. General and abdominal obesity parameters and their combination in relation to mortality: a systematic review and meta-regression analysis. Eur J Clin Nutr. 2013;67:573-85.

12. Emerging Risk Factors C, Wormser D, Kaptoge S, et al. Separate and combined associations of body-mass index and abdominal adiposity with cardiovascular disease: collaborative analysis of 58 prospective studies. Lancet. 2011;377:1085-95.

13. Jee SH, Sull JW, Park J, et al. Body-mass index and mortality in Korean men and women. N Eng J Med. 2006;355:779-87.

14. Vazquez G, Duval S, Jacobs DR Jr., Silventoinen K. Comparison of body mass index, waist circumference, and waist/hip ratio in predicting incident diabetes: a meta-analysis. Epidemiol Rev. 2007;29:115-28.

15. Han TS, Bijnen FC, Lean ME, Seidell JC. Separate associations of waist and hip circumference with lifestyle factors. Int J Epidemiol. 1998;27:422-30

16. Connor Gorber S, Tremblay M, Moher D, Gorber B. A comparison of direct vs. self-report measures for assessing height, weight and body mass index: a systematic review. Obes Rev. 2007;8:307-26.

17. Smith GD, Ebrahim S. 'Mendelian randomization': can genetic epidemiology contribute to understanding environmental determinants of disease? Int J Epidemiol. 2003;32:1-22.

18. Lawlor DA, Harbord RM, Sterne JA, Timpson N, Davey Smith G. Mendelian randomization: using genes as instruments for making causal inferences in epidemiology. Stat Med. 2008;27:1133-63.

19. Emdin CA, Khera AV, Natarajan P, et al. Genetic association of waist-to-hip ratio with cardiometabolic traits, type 2 diabetes, and coronary heart disease. JAMA. 2017;317:626-34.

20. Dale CE, Fatemifar G, Palmer TM, et al. Causal associations of adiposity and body fat distribution with coronary heart disease, stroke subtypes, and type 2 diabetes mellitus: a mendelian randomization analysis. Circulation. 2017;135:2373-88.

21. Hagg S, Fall T, Ploner A, et al. Adiposity as a cause of cardiovascular disease: a Mendelian randomization study. Int J Epidemiol. 2015;44:578-86.

22. Locke AE, Kahali B, Berndt SI, et al. Genetic studies of body mass index yield new insights for obesity biology. Nature. 2015;518:197-206.

23. Shungin D, Winkler TW, Croteau-Chonka DC, et al. New genetic loci link adipose and insulin biology to body fat distribution. Nature. 2015;518:187-96.

24. Nikpay M, Goel A, Won HH, et al. A comprehensive 1,000 Genomes-based genome-wide association meta-analysis of coronary artery disease. Nat Genet. 2015;47:1121-30.

25. Schunkert H, Konig IR, Kathiresan S, et al. Large-scale association analysis identifies 13 new susceptibility loci for coronary artery disease. Nat Genet. 2011;43:333-8. 
26. Hartwig FP, Davies NM, Hemani G, Davey Smith G. Two-sample Mendelian randomization: avoiding the downsides of a powerful, widely applicable but potentially fallible technique. Int $\mathrm{J}$ Epidemiol. 2016;45:1717-26.

27. Solovieff N, Cotsapas C, Lee PH, Purcell SM, Smoller JW. Pleiotropy in complex traits: challenges and strategies. Nat Rev Genet. 2013;14:483-95.

28. Cotsapas C, Voight BF, Rossin E, et al. Pervasive sharing of genetic effects in autoimmune disease. PLoS Genet. 2011;7: e1002254.

29. Global Lipids Genetics C, Willer CJ, Schmidt EM, et al. Discovery and refinement of loci associated with lipid levels. Nat Genet. 2013;45:1274-83.

30. International Consortium for Blood Pressure Genome-Wide Association S, Ehret GB, Munroe PB, et al. Genetic variants in novel pathways influence blood pressure and cardiovascular disease risk. Nature. 2011;478:103-9.

31. Ross S, Gerstein HC, Eikelboom J, Anand SS, Yusuf S, Pare G. Mendelian randomization analysis supports the causal role of dysglycaemia and diabetes in the risk of coronary artery disease. Eur Heart J. 2015;36:1454-62.

32. DIAbetes Genetics Replication And Meta-analysis (DIAGRAM) Consortium, Asian Genetic Epidemiology Network Type 2 Diabetes Consortium, et al. Genome-wide trans-ancestry metaanalysis provides insight into the genetic architecture of type 2 diabetes susceptibility. Nat Genet. 2014;46:234-44..

33. Bowden J, Davey Smith G, Burgess S. Mendelian randomization with invalid instruments: effect estimation and bias detection through Egger regression. Int J Epidemiol. 2015;44:512-25.

34. Burgess S, Butterworth A, Thompson SG. Mendelian randomization analysis with multiple genetic variants using summarized data. Genet Epidemiol. 2013;37:658-65.

35. Jarvis D, Mitchell JS, Law PJ, et al. Mendelian randomisation analysis strongly implicates adiposity with risk of developing colorectal cancer. Br J Cancer. 2016;115:266-72.

36. Mokry LE, Ross S, Timpson NJ, Sawcer S, Davey Smith G, Richards JB. Obesity and multiple sclerosis: a mendelian randomization study. PLoS Med. 2016;13:e1002053.

37. Ahmad OS, Leong A, Miller JA, et al. A Mendelian randomization study of the effect of type-2 diabetes and glycemic traits on bone mineral density. J Bone Miner Res. 2016;32:1072-81.

38. Bowden J, Davey Smith G, Haycock PC, Burgess S. Consistent estimation in mendelian randomization with some invalid instruments using a weighted median estimator. Genet Epidemiol. 2016;40:304-14.

39. Fall T, Hagg S, Magi R, et al. The role of adiposity in cardiometabolic traits: a Mendelian randomization analysis. PLoS Med. 2013;10:e1001474.
40. Nordestgaard BG, Palmer TM, Benn M, et al. The effect of elevated body mass index on ischemic heart disease risk: causal estimates from a Mendelian randomisation approach. PLoS Med. 2012;9:e1001212.

41. Yan LL, Daviglus ML, Liu K, et al. Midlife body mass index and hospitalization and mortality in older age. JAMA. 2006;295:190-8.

42. Mann DM, Lee J, Liao Y, Natarajan S. Independent effect and population impact of obesity on fatal coronary heart disease in adults. Prev Med. 2006;42:66-72.

43. Festa A, D'Agostino R Jr., Howard G, Mykkanen L, Tracy RP, Haffner SM. Chronic subclinical inflammation as part of the insulin resistance syndrome: the Insulin Resistance Atherosclerosis Study (IRAS). Circulation. 2000;102:42-7.

44. Yao L, Herlea-Pana O, Heuser-Baker J, Chen Y, Barlic-Dicen J. Roles of the chemokine system in development of obesity, insulin resistance, and cardiovascular disease. J Immunol Res. 2014;2014:181450.

45. Bhatta A, Yao L, Xu Z, et al. Obesity-induced vascular dysfunction and arterial stiffening requires endothelial cell arginase 1 . Cardiovasc Res. 2017;113:1664-76.

46. Sharma AM, Engeli S, Pischon T. New developments in mechanisms of obesity-induced hypertension: role of adipose tissue. Curr Hypertens Rep. 2001;3:152-6.

47. Wong CY, O'Moore-Sullivan T, Leano R, Byrne N, Beller E, Marwick TH. Alterations of left ventricular myocardial characteristics associated with obesity. Circulation. 2004;110:3081-7.

48. Pischon T, Boeing H, Hoffmann K, et al. General and abdominal adiposity and risk of death in Europe. $\mathrm{N}$ Eng $\mathrm{J}$ Med. 2008;359:2105-20.

49. Despres JP. Body fat distribution and risk of cardiovascular disease: an update. Circulation. 2012;126:1301-13.

50. Ng M, Fleming $\mathrm{T}$, Robinson $\mathrm{M}$, et al. Global, regional, and national prevalence of overweight and obesity in children and adults during 1980-2013: a systematic analysis for the Global Burden of Disease Study 2013. Lancet. 2014;384:766-81.

51. Lv WQ, Zhang $X$, Zhang $Q$, et al. Novel common variants associated with body mass index and coronary artery disease detected using a pleiotropic cFDR method. J Mol Cell Cardiol. 2017;112:1-7.

52. Chen Y, Copeland WK, Vedanthan R, et al. Association between body mass index and cardiovascular disease mortality in east Asians and south Asians: pooled analysis of prospective data from the Asia Cohort Consortium. BMJ. 2013;347:f5446.

53. Prospective Studies C, Whitlock G, Lewington S, et al. Bodymass index and cause-specific mortality in 900000 adults: collaborative analyses of 57 prospective studies. Lancet. 2009;373:1083-96. 